\title{
Seropositivity of SARS-CoV-2 IgG Antibody among People in Dhaka City during the Prevaccination Period
}

\author{
Zabed Bin Ahmed (D), Mamudul Hasan Razu (D), Fatema Akter (D), Md. Raisul Islam Rabby (D), \\ Pranab Karmaker $(D$, and Mala Khan
}

Division of Life Science, Bangladesh Reference Institute for Chemical Measurements (BRiCM), Dr. Qudrat-e-Khuda Road,

Correspondence should be addressed to Mala Khan; bricmdg@yahoo.com

Received 30 September 2021; Revised 9 November 2021; Accepted 7 January 2022; Published 27 January 2022

Academic Editor: Italo F. Angelillo

Copyright (c) 2022 Zabed Bin Ahmed et al. This is an open access article distributed under the Creative Commons Attribution License, which permits unrestricted use, distribution, and reproduction in any medium, provided the original work is properly cited.

\begin{abstract}
Severe acute respiratory syndrome coronavirus 2 (SARS-CoV-2) immunoglobulin G (IgG) detection can be an effective complementary tool to the reverse transcription-polymerase chain reaction (RT-PCR) test in estimating the true burden of coronavirus diseases 2019 (COVID-19) and can serve as baseline data, especially after the roll-out of vaccines against SARSCoV-2. In this study, we aim to determine the seropositivity of SARS-CoV-2 IgG among people in Dhaka, Bangladesh. Volunteers, mostly asymptomatic people from Dhaka, were enrolled between October 2020 and February 2021. After obtaining participants' signed consents, blood samples were tested for SARS-CoV-2 IgG antibody, following the standard protocol of testing within 72 hours of collection. SARS-CoV-2 IgG was positive in $42 \%(101 / 239)$ of the cases. No difference was observed in terms of IgG positivity and IgG levels when stratified by age, gender, and blood group. However, RT-PCRpositive cases presented higher IgG levels compared to RT-PCR-negative/RT-PCR-not performed cases. SARS-CoV-2 IgG was found in 31\% (32/102) and 28\% (19/67) of RT-PCR-negative and RT-PCR-not performed cases, respectively. For RTPCR-positive but SARS-CoV-2 IgG-negative cases $(n=13)$, the average time gap between the RT-PCR and SARS-CoV-2 IgG tests of six months indicates a gradual reduction of IgG. Eight cases for which samples were tested at two time points, three months apart, showed presented a decline in IgG levels with time (median IgG index of 2.55 in the first sample versus 1.22 in the second sample). Our findings reveal that several mild/asymptomatic cases that were RT-PCR-negative/not tested exist in the community, and $\operatorname{IgG}$ levels reduce in the human body over time.
\end{abstract}

\section{Introduction}

Coronavirus disease 2019 (COVID-19), first reported on December 30, 2019, in Wuhan Province of China, spreads rapidly worldwide, leading the World Health Organization (WHO) to declare it a pandemic on March 11, 2020 [1]. The first case of COVID-19 in Bangladesh was reported on March 8, 2020. Till April 26, 2021, 745,322 COVID-19 cases have been detected with 11,053 deaths. Thus, the overall prevalence of COVID-19 was $13.9 \%$, with a case fatality rate of $1.48 \%$ [2]. Managing this pandemic entails halting the virus transmission or developing immunity among the masses against SARS-CoV-2 through vaccination programs. To reduce virus transmission, various steps such as hand sanitization, wearing of face masks, and social distancing have been enforced throughout the country. However, for a more sustainable and practical solution to address this pandemic, the government has introduced a vaccine in the country, which has resulted in several policy measures, such as contingency plans, vaccine introduction, and identification of priority groups for vaccination.

An understanding of the true extent of the disease in the country is critical to guide and implement these public health measures. Bangladesh, like most countries, opted for the reverse transcription-polymerase chain reaction- (RTPCR-) based screening of only symptomatic patients. Despite this, the number of tests has been less than adequate. The detection of asymptomatic patients, their potential to 
TABLE 1: Demographic characteristics of the participants.

\begin{tabular}{lcc}
\hline Characteristics & Frequency & Percentage \\
\hline Age & 67 & \\
$\leq 30$ y & 60 & $28.0 \%$ \\
$31-40$ years & 36 & $25.1 \%$ \\
$41-50$ years & 42 & $15.1 \%$ \\
$51-60$ years & 34 & $17.6 \%$ \\
$>60$ years & & $14.2 \%$ \\
Gender & 196 & \\
Male & 43 & $82.0 \%$ \\
Female & & $18.0 \%$ \\
Blood group & 61 & \\
A+ & 67 & $29.6 \%$ \\
B+ & 49 & $32.5 \%$ \\
O+ & 29 & $23.8 \%$ \\
Others & 33 & $14.1 \%$ \\
Unknown & & $13.8 \%$ \\
Symptoms & 18 & \\
Yes & 221 & $7.5 \%$ \\
No & & $92.5 \%$ \\
RT-PCR & 67 & $28.0 \%$ \\
Positive & 94 & $39.3 \%$ \\
Negative & 67 & \\
Not performed & 11 & \\
Missing & & \\
\hline
\end{tabular}

spread the virus, and an inadequate number of tests indicate that the data generated from the country might be underestimated, and the true burden of the virus is much greater. According to the WHO, immunoglobulin G (IgG) and immunoglobulin $\mathrm{M}$ (IgM) surveillance against COVID-19 can help infer the extent of COVID-19 infection in the population [3].

Although RT-PCR is very sensitive and has been the mainstay for the detection of COVID-19 globally, the technique has its limitations from the policymaking perspective. The RT-PCR test is sensitive in terms of technical expertise, cost, transportation, and storage. The sensitivity of RT-PCRbased detection also depends on the timing of specimen collection [4]. As RT-PCR detects SARS-CoV-2 only in its active stages, this method is largely ineffective in detecting previous viral exposures [5].

Irrespective of the manifestation of symptoms, the human body produces antibodies in response to infections caused by various pathogens, including viruses. Given its relative simplicity compared to complex techniques such as RT-PCR, antigen/antibody test kits have been deployed, especially in epidemiological studies, as reliable instruments to measure the prevalence and patterns of infectious diseases [6]. Detection of antibodies, especially IgG-which is produced after two weeks of SARS-CoV-2 exposure and persists after the clearance of the infection-against SARS-CoV-2 can be an excellent indicator of previous, recovered, and undetected infections [7]. Therefore, it can effectively com- plement the RT-PCR test in understanding the true burden of COVID-19 in a country.

Despite its potential use in determining COVID-19 prevalence and addressing other scientific questions, data available on the seroprevalence of COVID-19 are mostly from developed countries, which differ from South Asian countries such as Bangladesh in terms of factors including infrastructure, culture, socioeconomic conditions, and careseeking behaviors. With the vaccine against SARS-CoV-2 already introduced in the country, understanding the level of antibodies produced in response to the vaccine during the postvaccine period is critical. However, the absence of relevant data during the prevaccine period renders the understanding of the impact and dynamics of the vaccine difficult. Therefore, in this study, we report the baseline data on the seropositivity and level of IgG among people in Dhaka during the prevaccination period.

\section{Methods}

We enrolled volunteers, mostly from Dhaka, with or without symptoms of COVID-19 infection between October 2020 and February 2021. COVID-19 cases were defined as cases that were confirmed through the RT-PCR test. After obtaining signed consent, the volunteers were asked to complete a questionnaire to collect information. The questionnaire contains participant's demographic information (age, gender, area of living, blood group. etc.) and clinical information (presenting symptoms if any, history of comorbidity, etc.). Following the standard protocol, blood samples of $3 \mathrm{ml}$ each were collected from the participants in a clot activator tube. The blood was centrifuged at 2,000 rpm for $5 \mathrm{~min}$ to separate the serum. The serum was separated and immediately stored at $-20^{\circ} \mathrm{C}$ if not processed the same day. All blood serum samples were tested within $72 \mathrm{~h}$ of collection.

The sera were tested for SARS-CoV-2 IgG antibodies using the commercially available enzyme-linked immunoassay (ELISA) kit, ErbaLisa COVID-19 IgG. The ErbaLisa COVID-19 IgG kit is a European CE marked ELISA test kit that allows semiquantitative detection of SARS-CoV-2 IgG antibody in human serum samples with declared relatively high sensitivity and specificity of $98.3 \%$ and $98.1 \%$, respectively [8]. All serum samples were tested according to the manufacturer's instructions. Briefly, $10 \mu \mathrm{L}$ of serum samples was diluted with $200 \mu \mathrm{L}$ commercially available sample diluent (1:20 dilution); $100 \mu \mathrm{L}$ of diluted samples was dispensed in specific microliter plates, and the subsequent steps were followed following the manufacturer's guidelines. The IgG antibody index was calculated to understand the level of IgG in the serum sample as per manufacturer's instruction: antibody index $<0.9$ was considered as negative, and $\geq 0.9$ was considered as positive.

We collected sequential samples for eight cases that presented positive results in the RT-PCR test at two time points: one between 14 and 30 days of the onset of infection and the other after 2 months of the first sample. These cases presented negative COVID-19 results in the RT-PCR tests between the periods of two sequential samples. 


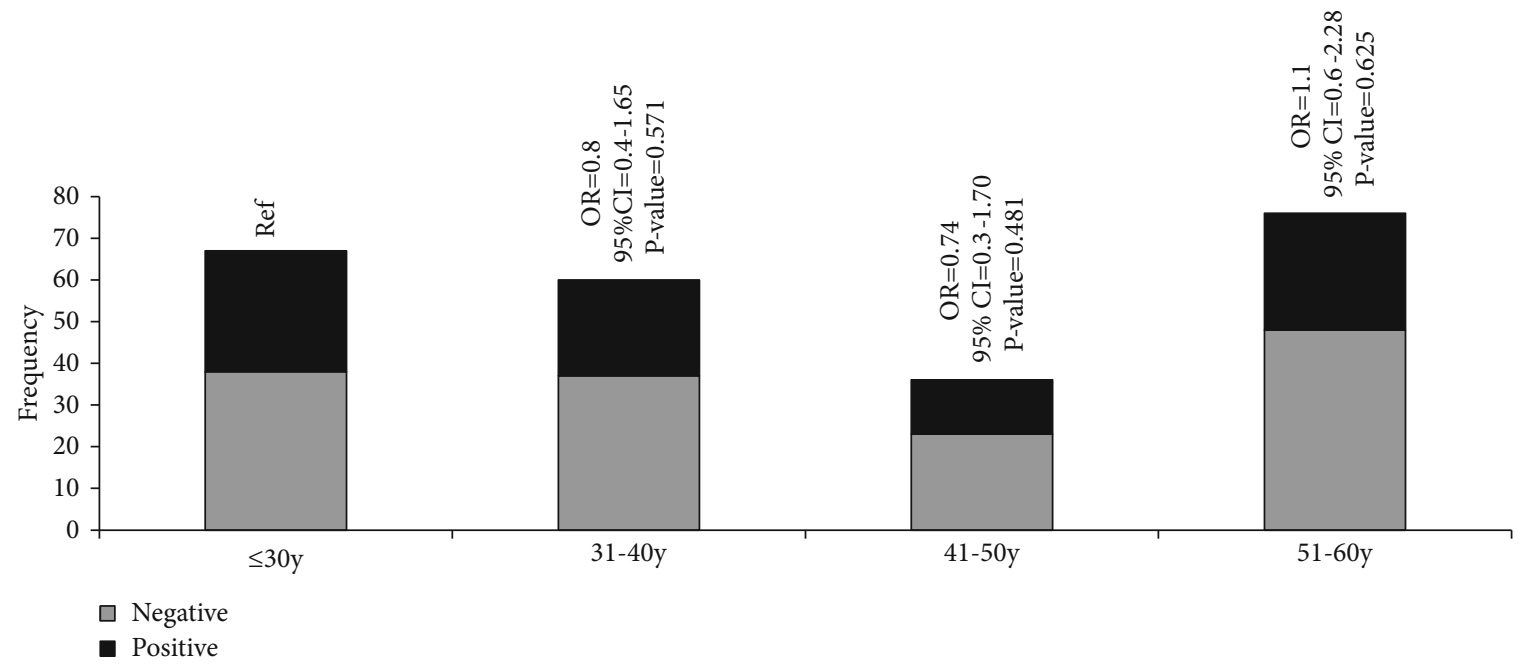

(a)

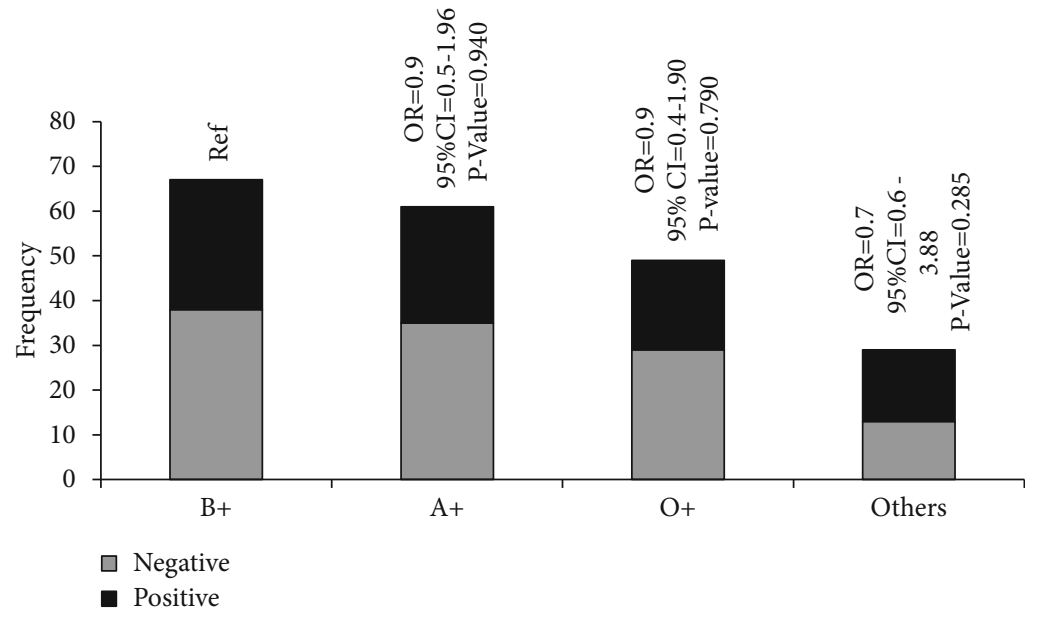

(b)

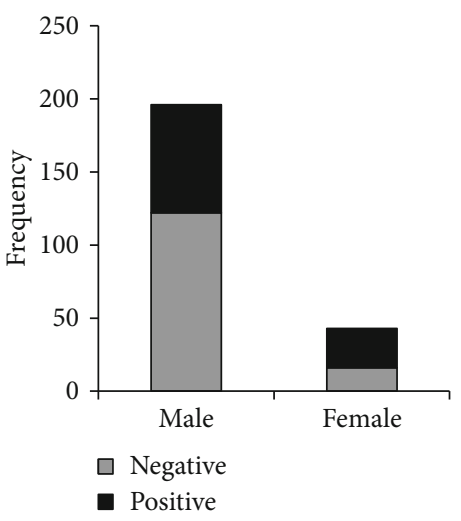

(c)

FIGURE 1: SARS-CoV-2 IgG seropositivity by (a) age group, (b) blood group, and (c) gender.

TABLE 2: Comparison of RT-PCR and SARS-CoV-2 IgG results $(n=227)$.

\begin{tabular}{lccc}
\hline \multirow{2}{*}{ RT-PCR result } & \multicolumn{2}{c}{ SARS-CoV-2 IgG result } & \multirow{2}{*}{ Total } \\
& Negative (\%) & Positive (\%) & \\
\hline Negative & $70(68.6)$ & $32(31.4)$ & 102 \\
Positive & $13(22.4)$ & $45(77.6)$ & 58 \\
Not performed & $48(71.6)$ & $19(28.4)$ & 67 \\
Total & 131 & 96 & 227 \\
\hline
\end{tabular}

Multinomial logistic regression analysis was performed to compare SARS-CoV-2 IgG positivity among the different subgroups. IgG levels among different subgroups were compared using the Wilcoxon rank-sum test. All analyses were performed using the Stata software version 13.

\section{Results}

A total of 239 serum samples were collected between October 2020 and February 2021. The mean age of the patients was $42 \pm 14.5$ years (range: $5-80$ ) with a male-female ratio of 4.5 . The age of the majority of the participants (53\%) was $<40$ years, with the highest share of participants (27.7\%) being in the 20-30 years' age group. The lowest share of participants (14.3\%) was in the age group of $>60$ years. The blood group was available for 206 cases, of which $\mathrm{B}$ positive was the most common (32.5\%), followed by A positive $(29.6 \%)$ and O positive $(23.8 \%)$. The demographic characteristics of the participants are presented in Table 1.

Overall, the seropositivity of the SARS-CoV-2 IgG antibody was found to be $42.2 \%$ (101/239). When stratified by age, gender, and blood group, no difference was observed in terms of IgG positivity (see Figure 1).

Cases for which both RT-PCR and SARS-CoV-2 IgG results were available numbered 227. Of the 102 cases that were reported as RT-PCR negative, 31\% (32/102) were found to be positive for IgG. Alternatively, in 22.48\% (13/ 58) of the RT-PCR-positive cases, no IgG antibody was detected. Out of these 13 cases, the duration between COVID-19 infection and SARS-CoV-2 IgG was available for 7 cases. The average duration between RT-PCR 


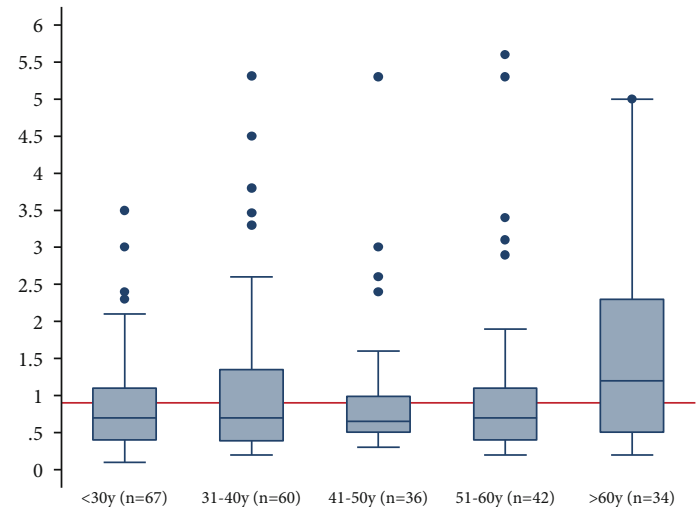

(a)

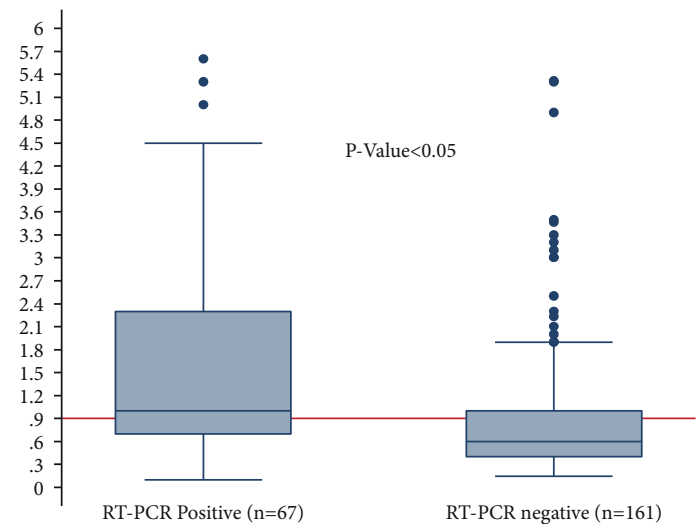

(c)

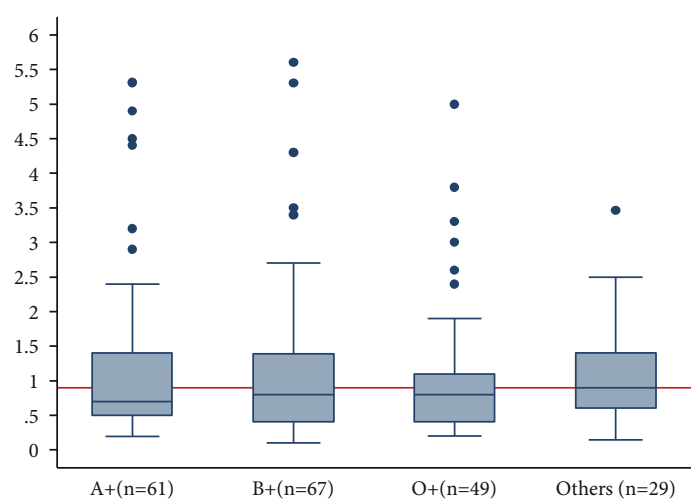

(b)

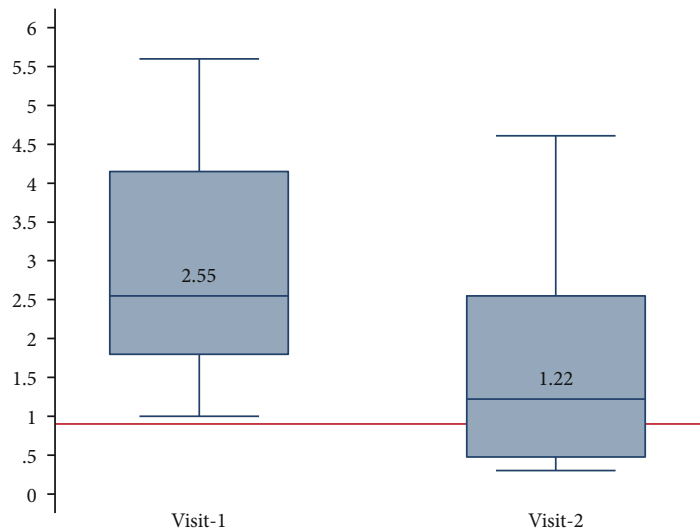

(d)

FIgURe 2: SARS-CoV-2 IgG index by (a) age group, (b) blood group, (c) RT-PCR results, and (d) at two time points.

confirmed cases and the SARS-CoV-2 IgG test was 5 months (range: 3-6 months) for these 7 cases. SARS-CoV-2 IgG was found in $28 \%(19 / 67)$ of the patients who were never tested by RT-PCR (see Table 2 ).

The level of antibodies observed among the different age groups and blood groups did not differ significantly (Figures 2(a) and 2(b)). However, the antibody level of IgG in participants with RT-PCR-positive cases was found to be significantly higher: the median IgG index of RT-PCRpositive and-negative cases was 1.0 and 0.6 , respectively (Figure 2(c)). Of the eight cases from which specimens were collected at two time points, all presented a gradual reduction in IgG levels over time. The median IgG index at the first and second visit was 2.55 and 1.22, respectively (Figure 2(d)). However, the degree of reduction in IgG levels varied among the individuals (data not shown).

\section{Discussion}

The seropositivity of IgG antibodies among the samples tested for our study, 6 months after the first detected case in the country, was found to be $42 \%$. This is much higher than the community seroprevalence reported in other parts of the world: $2.8 \%$ in California, USA [9], 7.6\% in Daegu, Korea [10], 5\% in Spain [11], a comparable $22-33 \%$ in Iran [12], and $25.41 \%$ in Niger State, Nigeria [13]. This proportion indicates that the true burden of COVID-19 in Bangla- desh might be much greater than that estimated by the RTPCR-based detection method. This may be due to several mild/asymptomatic/recovered cases that were never tested using RT-PCR for various reasons or because of the ineffectiveness of RT-PCR in detecting previous infections. In terms of SARS-CoV-2 IgG seropositivity, no significant difference was found when stratified by the age and blood group. The male population was predominant in our study. Hence, we could not compare seropositivity and IgG index for SARS-CoV-2 in terms of gender.

Almost one-third (31.4\%) of RT-PCR-negative cases were found positive for SARS-CoV-2 IgG, which further corroborates the assumption of several cases missed by RT-PCR-based techniques. Even in cases that were never tested by RT-PCR, approximately $28 \%$ were found to be positive for IgG. This indicates the underestimation of the burden of COVID-19 through RT-PCR, which is in concordance with the findings from other parts of the world [9]. For the RT-PCR-positive cases, we found 77.6\% IgG positivity after 3-4 weeks of COVID-19 symptom onset. This is higher than the $45 \%$ IgG positivity among cases of RT-PCR-positive asymptomatic cases reported by Shirin et al. [14]. Interestingly, out of 58 RT-PCR-positive cases, we did not find detectable IgG antibodies in 13 cases. To investigate whether the long duration between the RT-PCR test and COVID-19 IgG may cause this, a further analysis among seven such cases (RT-PCR date was not available 
for the rest of the cases) demonstrated that the average duration between the RT-PCR confirmation and IgG test of these cases was 5 months (range: 3-6 months). By contrast, out of the remaining 45 RT-PCR-positive cases (which were IgGpositive), RT-PCR data were available for 24 cases. The average duration between the RT-PCR confirmation and IgG test of these 24 cases was 3.8 months (range: $1-7$ months). This might indicate the possibility that the level of IgG reduces with time, and by the time these cases were tested, their IgG levels were below the detection level.

To test this possibility of reducing IgG levels even further with time, we randomly selected eight IgG-positive cases to examine the level of IgG at two time points: first, serum within 14-30 days of RT-PCR confirmation and second, serum 3 months after the first sample. We found that the median IgG index of these cases reduced with time: 2.55 in the first sample vs. 1.22 in the second sample. However, the reduction in IgG levels with time varied among individuals. This finding is similar to reports published in other parts of the world: Iyer et al. reported slow decay of SARSCoV-2 IgG through 90 days after the onset of symptoms [7]. Long et al. also found a decline in SARS-CoV-2 IgG two weeks after discharge from the hospital [15]. Similar findings of a decrease in IgG levels with time were also reported by Ibarrondo et al. [16] and Campos et al. [17]. However, a later study reported that despite declining, IgG levels were detectable for more than six months.

The study has the following limitations: (1) the sample size was relatively small and participants enrolled voluntarily, which might not represent the true population of the country. Moreover, the study participants were heavily dominated by males. (2) For the longitudinal cohort to study IgG levels over time, we enrolled only eight cases and analyzed them for two time points. (3) We only checked SARSCoV-2 IgG in this study. IgM and IgA and neutralizing antibody analysis as a whole are required for a comprehensive understanding of the dynamics of the SARS-CoV-2 antibody.

\section{Conclusions}

This is one of the first studies to shed light on SARS-CoV-2 IgG seropositivity in Bangladesh. Our results indicated that the seropositivity of IgG among the participants tested was $42 \%$, and the IgG positivity did not differ in terms of age, gender, and blood group. We also revealed several mild/ asymptomatic patients in the community, who either tested negative in the RT-PCR test or never tested for SARS-CoV2. Our findings also demonstrated that with time, the level of IgG reduces within the human body. As Bangladesh recently introduced a vaccine against COVID-19, further comprehensive SARS-CoV-2 antibody analysis during the postvaccine period with large sample size is required to guide policy decisions.

\section{Data Availability}

The data used to support the findings of this study are included in this article.

\section{Ethical Approval}

The study was approved by the Ethics Review Committee of the Bangladesh Council of Scientific and Industrial Research (BCSIR), ref no-39.11.0000.003.37.005.20/12, date09.24.2020.

\section{Consent}

Written consent was obtained from all the participants. Data were restricted to authorized persons only and analyzed anonymously.

\section{Conflicts of Interest}

The authors declare that there are no conflicts of interest regarding the publication of this paper.

\section{Authors' Contributions}

ZA analyzed the samples, monitored the study progress, analyzed the data, and wrote the manuscript. MR analyzed the samples, monitored the study progress, managed the logistics, and reviewed the manuscript. FA and MRI performed sample analysis. PK reviewed the manuscript. MK led and directed the study, was a major contributor to writing the manuscript, and monitored the progress of the entire study.

\section{Acknowledgments}

We thank Mr. Md. Enamul Haque and Mr. Sattya Ranjan, who helped with blood collection, serum separation, and study management. We are grateful to the study committee and volunteers for agreeing to participate in the study and the study staff members for their diligent efforts. This study was funded by the Ministry Science and Technology, People's Republic of Bangladesh.

\section{References}

[1] WHO, Director-General's opening remarks at the media briefing on COVID-19-March 11, 2020, April 27, 2021, https:// www.who.int/director-general/speeches/detail/who-directorgeneral-s-opening-remarks-at-the-media-briefing-on-covid19-11-march-2020.

[2] Covid 19 dashboard, 2020, April 26, 2021, https://corona.gov .bd/?gclid=Cj0KCQjwppSEBhCGARIsANIs4p7b6dJyN3Sjg XB_OJZY32oYpThWAQcNCZDi4bPZNNLc5xrN7jjLO0aAn ObEALw_wcB.

[3] World Health Organization, Population-based age-stratified seroepidemiological investigation protocol for coronavirus 2019 (COVID-19) infection, 2020, April 27, 2021, https:// www.who.int/publications/i/item/WHO-2019-nCoVSeroepidemiology-2020.2.

[4] A. Afzal, "Molecular diagnostic technologies for COVID-19: limitations and challenges," Journal of Advanced Research, vol. 26, pp. 149-159, 2020.

[5] K. K.-W. To, O. T. Y. Tsang, W. S. Leung et al., "Temporal profiles of viral load in posterior oropharyngeal saliva samples and serum antibody responses during infection by SARS-CoV-2: 
an observational cohort study," The Lancet Infectious Diseases, vol. 20, no. 5, pp. 565-574, 2020.

[6] J. E. Bryant, A. S. Azman, M. J. Ferrari et al., "Serology for SARS-CoV-2: apprehensions, opportunities and the path forward," Sci Immunol, vol. 5, no. 47, article eabc6347, 2020.

[7] A. S. Iyer, F. K. Jones, A. Nodoushani et al., "Persistence and decay of human antibody responses to the receptor binding domain of SARS-CoV-2 spike protein in COVID-19 patients," Science immunology, vol. 5, no. 52, pp. 1-12, 2020.

[8] ErbaLisa, COVID-19 IgG CE-marked Enzyme Immunoassay (ELISA) kit for the detection of IgG antibodies to SARS-CoV-2 in human serum, 2021, April 26, 2021, https:// erbalisacovid19.erbamannheim.com/getattachment/Home/ image-content/Covid/Erba-COVID-19-IgG-A4-Flyer-v3.pdf .aspx?lang=en-US\&ext=.pdf.

[9] E. Bendavid, B. Mulaney, N. Sood et al., COVID-19 antibody seroprevalence in Santa Clara County, California, medRxiv, 2020.

[10] S. K. Song, D. H. Lee, J. H. Nam et al., "IgG seroprevalence of COVID-19 among individuals without a history of the coronavirus disease infection in Daegu, Korea," Journal of Korean medical science, vol. 35, no. 29, pp. 1-8, 2020.

[11] M. Pollán, B. Pérez-Gómez, R. Pastor-Barriuso et al., "Prevalence of SARS-CoV-2 in Spain (ENE-COVID): a nationwide, population-based seroepidemiological study," Lancet, vol. 396, no. 10250, pp. 535-544, 2020.

[12] M. Shakiba, S. S. H. Nazari, F. Mehrabian, S. M. Rezvani, Z. Ghasempour, and A. Heidarzadeh, Seroprevalence of COVID-19 virus infection in Guilan province, Iran, medRxiv, 2020.

[13] H. Majiya, M. Aliyu-Paiko, V. T. Balogu et al., Seroprevalence of COVID-19 in Niger state, medRxiv, 2020.

[14] T. Shirin, T. R. Bhuiyan, R. C. Charles et al., "Antibody responses after COVID-19 infection in patients who are mildly symptomatic or asymptomatic in Bangladesh," International Journal of Infectious Diseases, vol. 101, pp. 220-225, 2020.

[15] Q. X. Long, X. J. Tang, Q. L. Shi et al., "Clinical and immunological assessment of asymptomatic SARS-CoV-2 infections," Nature Medicine, vol. 26, no. 8, pp. 1200-1204, 2020.

[16] F. J. Ibarrondo, J. A. Fulcher, D. Goodman-Meza et al., "Rapid decay of anti-SARS-CoV-2 antibodies in persons with mild COVID-19," The New England Journal of Medicine, vol. 383, no. 11, pp. 1085-1087, 2020.

[17] P. Figueiredo-Campos, B. Blankenhaus, C. Mota et al., "Seroprevalence of anti-SARS-CoV-2 antibodies in COVID-19 patients and healthy volunteers up to 6 months post disease onset," European Journal of Immunology, vol. 50, no. 12, pp. 2025-2040, 2020. 